\title{
DIÁLOGOS INTRA E INTEREPISTÉMICOS EN CUSI CUSI ACERCA DEL TIEMPO. UN EJERCICIO REFLEXIVO
}

\author{
Intra and Inter epistemic Dialogues About Time in Cusi Cusi. A Reflective Exercise
}

\author{
JOSÉ MARÍA VAQUER*, BÁRBARA SOFÍA CARBONI**, FACUNDO PETIT*** \\ \& MARTINA DI TULLIO****
}

Fecha de recepción: 05 de junio de 2020 - Fecha de aprobación: 07 de noviembre de 2020

\section{Resumen}

En este trabajo presentamos un ejercicio teórico-práctico de antropología/arqueología dialógica, en el que proponemos repensar críticamente cómo se construyen las narrativas arqueológicas sobre el pasado. El ejercicio consiste en utilizar el análisis de una serie de entrevistas etnográficas realizadas en Cusi Cusi (Rinconada, Jujuy), en las cuales buscamos interpretar las categorías de tiempo y espacio en la lógica de sus habitantes y ponerlas en diálogo con nuestras lógicas científicas. Utilizando las entrevistas como expresiones de las vivencias que permiten interpretar las categorías de tiempo y espacio y sus relaciones, el objetivo es desalentar una narrativa totalizante, donde solo está contenida la voz científica que subsume a las demás, para considerar las narrativas sobre el pasado en cuanto resultado de distintas lógicas. A la vez, el trabajo permite repensar cómo se construyen las prácticas académicas y cuáles son sus efectos en la configuración epistemológica de los espacios y los sujetos.

Palabras clave: diálogo epistémico; arqueología dialógica; temporalidad; espacialidad; Cusi Cusi.

\begin{abstract}
In this work we present a theoretical-practical exercise in dialogical anthropology/archaeology, in which we propose to critically re-think how archaeological narratives of the past are constructed. This exercise consists in using the analysis of a series of ethnographic interviews carried out in Cusi Cusi (Rinconada, Jujuy) in which we attempt to interpret the categories of time and space that are present in their inhabitants's logic and to put them in dialog with our own scientific logics. In using the interviews as expressions of lived experiences that allow to interpret the categories of time and space and their relationships, the objective is to discourage a totalizing narrative, in which only the scientific voice that subsumes others is included, and to acknowledge the narratives of the past as the result of different logics. Moreover, this work allows to re-think how academic practices are constructed and their effects on the epistemological configuration of spaces and subjects.
\end{abstract}

Keywords: epistemic dialogue; dialogical archaeology; temporality; spatiality; Cusi Cusi.

\footnotetext{
* Dr. en Arqueología. Investigador Asistente del Consejo Nacional de Investigaciones Científicas y Técnicas (CONICET). Instituto de Arqueología, Facultad de Filosofía y Letras, Universidad de Buenos Aires, Buenos Aires, Argentina. Artículo enmarcado en el proyecto FILOCYT "Paisaje, memoria y territorio en Cusi Cusi (Rinconada, Jujuy): hacia un diálogo inter-epistémico" (FC 19-068), dirigido por el Dr. José María Vaquer y financiado por la Facultad de Filosofía y Letras de la Universidad de Buenos Aires. Correo-e: jmvaquer1977@gmail.com

** Estudiante de Ciencias Antropológicas, orientación Arqueología. Investigadora en formación en el Instituto de Arqueología, Facultad de Filosofía y Letras, Universidad de Buenos Aires, Buenos Aires, Argentina. Correo-e: bscarboni@gmail.com *** Dr. en Antropología. Becario doctoral del Consejo Nacional de Investigaciones Científicas y Técnicas (CONICET). Instituto de Ciencias Antropológicas, Facultad de Filosofía y Letras, Universidad de Buenos Aires, Buenos Aires, Argentina. Correo-e: facundo.petit@gmail.com

**** Estudiante de Ciencias Antropológicas, orientación Arqueología. Investigadora en formación en el Instituto de Arqueología, Facultad de Filosofía y Letras, Universidad de Buenos Aires, Buenos Aires, Argentina. Correo-e: ditulliomartina@gmail.com
} 


\section{Introducción}

El pasado ya sirve y abre el presente para ver las tres cuestiones: pasado, presente y futuro.

Fragmento de entrevista realizada a M. L.

El término quechua pallqa significa el encuentro de dos o más caminos que se unen. Siguiendo este espíritu, consideramos que nuestro trabajo en la región de Cusi Cusi debe ser pallqa. En el paisaje cuseño nos encontramos nosotros, equipo de arqueólogos(as) y antropólogos(as), y ellos, los comuneros y comuneras de Cusi Cusi. Arqueólogos(as) haciendo antropología y antropólogos(as) haciendo arqueología, desafiando y tensando los límites virtuales que separan a ambas disciplinas. Nos encontramos con el pasado, con el presente y con el futuro. $\mathrm{Y}$, sobre todo, nos encontramos con lógicas diversas a la hora de interpretar estas categorías. Las lógicas que tenemos nosotros, agentes en un campo académico, derivadas de las categorías de análisis producto de nuestros intereses científicos y nuestras posiciones en los diversos campos que ocupamos. A su vez, nos encontramos con las categorías propias de los cuseños, también producto de los campos que ocupa cada uno, de sus trayectorias sociales y biográficas.

Con respecto a los campos que ocupamos nosotros, es muy difícil trazar las líneas divisorias. ¿Dónde termina la antropología y empieza la arqueología? Nos parece que tales cuestiones derivan en discusiones estériles. La arqueología que realizamos es una arqueología por y para el presente. La cultura material del pasado es un fenómeno que transcurre en el presente. Las interpretaciones del pasado tienen un efecto práctico en el presente. A su vez, la antropo- logía nos aporta un elemento fundamental: las interpretaciones de los habitantes actuales con respecto al pasado, al presente y al futuro. Cuando insertamos a la arqueología y a la antropología en un continuum, y consideramos las relaciones entre el pasado, el presente y el futuro, las líneas divisorias se borran.

En esta oportunidad proponemos un ejercicio de antropología/arqueología dialógica, donde interpretamos las categorías de tiempo y espacio en Cusi Cusi y las ponemos en diálogo con las nuestras, en cuanto habitantes de "mundos de la vida" diferentes. El objetivo (y el desafío) es no totalizar con nuestra lógica las visiones del tiempo y el espacio de los cuseños, sino tomarlas en su alteridad. Totalizar una narrativa implica sacrificar lo individual por lo grupal, utilizar lo otro en provecho de lo uno, produciendo de esa manera una alienación de la singularidad (Levinas, 2002). El espíritu de este trabajo es deconstruir nuestro lugar de enunciación. Por lo tanto, el ejercicio que proponemos es de orden reflexivo para la arqueología y la construcción de narrativas sobre el pasado. Al ser un trabajo que busca el diálogo, hacia el final del texto no se encontrarán con una síntesis, sino con más preguntas que respuestas. Compartimos un punto de partida de un proceso deconstructivo de las narrativas arqueológicas totalizantes, un diálogo intra e interepistémico.

\section{Herramientas interpretativas}

En esta sección desarrollamos las herramientas que utilizamos para interpretar la temporalidad propia y la de los cuseños. Para ello, proponemos un recorrido por la fenomenología aplicada a la descripción del "mundo de la vida" (Husserl, 2008; Schütz \& Luckmann, 2009) para sentar las bases de las interpretaciones posteriores. Sostenemos 
que toda interpretación se encuentra anclada en el "mundo de la vida" como horizonte que dona el sentido a los diferentes campos sociales (Vaquer, 2018). En muchos casos los presupuestos que conforman este horizonte se encuentran implícitos, por lo que es necesario reconocerlos para generar interpretaciones críticas. A su vez, la fenomenología del "mundo de la vida" nos permite abordar los presupuestos que dan sentido a las vivencias de los cuseños. Consideramos los mecanismos que describe la fenomenología como la base común de los esquemas que constituyen las diferentes cosmovisiones. Reconocer estas bases comunes es lo que permite abordar una etnografía que se centre en la diferencia entre nosotros y los cuseños.

La metodología etnográfica que utilizamos proviene de la "antropología de la experiencia" de Turner y Bruner (1986). El hilo conductor de esta sección es el concepto de vivencia, entendido como las experiencias de los agentes como se presentan en la conciencia. Para acceder a las vivencias es necesario que estas se transformen en expresiones, que pueden ser representaciones, performances, textos u objetivaciones de cualquier tipo (Bruner, 1986a). En este sentido, consideramos que las entrevistas etnográficas y su registro, tanto grabado como transcripto, son expresiones de las vivencias que permiten interpretar las categorías de tiempo y espacio y sus relaciones.

\section{Fenomenología del "mundo de la vida"}

La fenomenología fue concebida para atacar uno de los grandes problemas de la teoría del conocimiento occidental, que Husserl (1982) denominó "el problema de la trascendencia". Este problema implica que, desde el punto de vista del sujeto, solamente podemos estar seguros de los contenidos de nuestra conciencia. No ocurre lo mismo para el caso de los objetos que se encuentran fuera de la conciencia (ver Vaquer, 2015 y 2018, para una ampliación y aplicación del método fenomenológico). La fenomenología no se centra solamente en el objeto percibido, sino que considera los horizontes que lo rodean. En cualquier acto intencional, donde la conciencia se dirige a un objeto, siempre se encuentra presente el mundo en cuanto horizonte de fondo. En este sentido, el mundo se corresponde con todo lo que queda en estado de latencia en una percepción. Esto nos permite describir el "mundo de la vida" en términos del horizonte sobre el que se desarrolla la vida cotidiana de los agentes.

Schütz y Luckmann (2009) proponen que el "mundo de la vida" es todo lo que se da por sentado en el sentido común. Es la garantía de seguridad ontológica, todo lo que experimentamos como incuestionable. La explicitación y la comprensión del mundo se basan en un "acervo de experiencia previa", constituido por las experiencias propias inmediatas y las experiencias que transmiten los semejantes, padres, maestros, etc. Todas estas experiencias están incluidas en una cierta unidad que tiene la forma de un "acervo de conocimiento", el cual sirve como esquema de referencia para las acciones y las percepciones. El "mundo de la vida" es una experiencia grupal sedimentada que ha pasado la prueba y cuya validez no necesita ser examinada por los individuos. En la actitud natural -el sentido común- se toma conciencia del carácter deficiente del "acervo de conocimiento" únicamente si una experiencia nueva no se adecúa a lo que hasta ahora ha sido considerado como el esquema de referencia válido presupuesto. 
En consecuencia, todas las interpretaciones de la ciencia descansan en algún punto sobre el mundo de la vida del científico y sobre su "acervo de conocimiento". Nuestro objeto de estudio es el "mundo de la vida" de los otros. Por lo tanto, la interpretación implica que, desde nuestro "mundo de la vida" particular, con su "acervo de conocimiento" específico, abordemos un "mundo de la vida" ajeno y extraño (ver Wright, 1994, para una reflexión similar desde la etnografía).

\section{Antropología de la experiencia}

La antropología de la experiencia fue formulada inicialmente por Turner y Bruner (1986). La experiencia es considerada una relación directa entre los sujetos con aquello que se entiende como realidad. Dicha relación se da a través de los diferentes sentidos y conforma una suerte de unidad. De esta manera, el mundo se aprehende de manera relacional y se mantiene en el plano subjetivo. La experiencia inmanente de la conciencia se interpreta a partir de las expresiones, que son las "unidades estructuradas de la experiencia" (Bruner, 1986a, p. 7) o unidades socialmente construidas de significado. Lo que se cuenta de la realidad está atravesado por la experiencia, sea en relatos, monumentos, dibujos, modos de organización social. Es decir, todo lo que produce ese traspaso de lo individual a lo interpersonal está mediado por una primera interpretación.

La relación entre las expresiones y la experiencia es dialéctica y dialógica (Bruner, 1986a, p. 6). Así como la experiencia estructura las expresiones, las expresiones estructuran e interpelan la experiencia. La antropología de la experiencia nos brinda una serie de marcos conceptuales y metodológicos para atender las realidades de nuestros interlocutores. Sin embargo, el trabajo reflexivo no termina ahí. La etnografía se estructura como un género más, donde el proceso interpretativo es doble: la gente interpreta sus experiencias en formas de expresión, y nosotros, en el trabajo de campo, interpretamos esas expresiones en "nuestras historias sobre sus historias" (Bruner, 1986a, p. 10). Esta reflexión sobre la investigación misma es sintetizada por Bruner (1986b), en un trabajo de la misma compilación, bajo el concepto de "narrativa".

Es por esa razón que la antropología de la experiencia nos permite trabajar desde un triple proceso. En primer lugar, abordar las categorías del tiempo de los cuseños a partir de sus expresiones. En segundo lugar, realizar un análisis de las narrativas implícitas del trabajo científico, construidas en un doble plano, por lo articulado en el ámbito académico y lo vivido por el equipo de trabajo. En tercer lugar, atender a las relaciones entre ambas narrativas, interpretando las diferencias, pero también planteando las semejanzas, ya que "tanto el antropólogo como el informante nativo participan en el mismo sistema simbólico" (Bruner, 1986b, p. 150). Es este último punto el que pretendemos contextualizar en este artículo, entendiendo que, desde el momento en que las historias se comparten, pertenecen a un mismo universo narrativo. Ya no pertenecen ni a uno ni a otro, sino que se sitúan en el plano de lo dialógico, cuya interpretación es intercultural.

Cuando planteamos una antropología/ arqueología, lo hacemos desde una postura relacional, dialógica, que se abstenga de jerarquizar discursos y prácticas. En el plano local, es lo que Wright (1994, p. 365) denomina el "turno existencial de la experiencia". Plantea, 
así, una praxis etnográfica con énfasis en la existencia para el análisis de la experiencia, junto con las estructuras que la mediatizan, condicionan o determinan. De esta manera, el trabajo de campo supone un "desplazamiento ontológico" (Wright, 1994, p. 367), en el que el ser-en-el-mundo cambia de lugar y sufre modificaciones estructurales, creando al intersujeto: "una certeza existencial de que nuestro propio mundo, previamente sentido como dado, ahora se toma una posibilidad entre otras muchas, y que esa posibilidad se halla en relación heterárquica y no jerárquica con respecto a la de nuestros interlocutores" (Wright, 1994, p. 370). Este cuestionamiento existencial es la base del método que hemos desarrollado para la construcción de los datos y el análisis de las experiencias. Si bien la reflexividad se encuentra presente en toda práctica desarrollada por el equipo, aquí nos centramos en el "lugar antropológico" (Wright, 2005, p. 57), es decir, cómo se construye históricamente la práctica académica y sus efectos en la configuración epistemológica de los espacios y los sujetos.

\section{Metodología}

En un trabajo anterior (Vaquer, Petit \& Di Tullio, 2020) describimos el proceso a través del que se diagramó y realizó una serie de entrevistas a varios habitantes de Cusi Cusi a partir de su interés en desarrollar un museo comunitario. Por medio de la transcripción, acudimos a un análisis semiótico, en el que dividimos las referencias a la temporalidad en tres aspectos: semántico, pragmático y las referencias al espacio. Fue a partir de aquellos intercambios que nos surgieron interrogantes en torno a la concepción del tiempo en la experiencia de nuestros interlocutores. El presente artículo trabaja con inter- pretaciones sobre el mismo corpus de datos, pero haciendo foco en cuestiones diferentes. Cabría, entonces, una mayor contextualización de las entrevistas realizadas.

Como ya se mencionó, el objetivo de las preguntas consistió en generar una instancia dialógica para concebir un proyecto de museo que no partiera de nuestros presupuestos, sino que se realizara en conjunto con la comunidad. Se trata de una postura política explícita en nuestra participación con los proyectos del pueblo: no diseñar nada, sino hasta que dicha construcción se dé en conjunto, especialmente cuando el habitus académico es necesario en el lenguaje técnico requerido para la producción de este tipo de documentos.

Durante diciembre de 2016, se realizaron quince entrevistas, siguiendo el método etnográfico (Guber, 2011), a partir de una serie de preguntas y tópicos que estructuraron el diálogo. Los entrevistados representan una diversidad que es inherente al universo cuseño en lo relativo a cuestiones etarias, de género, de participación activa en la política institucional y de adscripción religiosa. Las preguntas apuntaron a conocer la valoración que se tiene del pasado en relación con el presente, elementos identitarios que podrían formar parte del futuro museo, y cuestiones de infraestructura y manejo del mismo.

El marco de la entrevista apuntó a crear un clima en el que cada interlocutor se sintiera cómodo para ahondar en diferentes temáticas, desde historias personales y del pueblo, sentimientos en torno al presente y discursos que tuvieron a la religión como protagonista. Contemplando el método propuesto por la antropología de la experiencia, todos estos registros fueron tomados como expresiones de la experiencia 
socialmente articulada de ser habitante de Cusi Cusi. Es por eso que, en cada vivencia relatada, con los modos de conjugación de las frases y las relaciones establecidas, fuimos obteniendo indicios acerca de la temporalidad y la espacialidad construidas socialmente por los cuseños. Sin embargo, no es sino en el análisis de las entrevistas, de la literatura y de nuestros diarios de campo que podremos llegar a saber si el pensamiento de los cuseños, social y espacialmente situado, se vincula con las experiencias narradas, en particular con la temporalidad.

El siguiente objetivo de este trabajo fue poner estas concepciones en diálogo con las propias del científico social. En este caso, seres urbanos, con intereses específicos y explícitos, y con una existencia que tiene sus bases en la tradición europea de pensamiento. Así, ese diálogo se instaura desde una relectura reflexiva de nuestros diarios de campo, del recuerdo de nuestras experiencias, de la construcción de una doble alteridad y de la revisión histórica de la tradición arqueológica y antropológica, a la manera de una etnografía de la práctica arqueológica y antropológica (Edgeworth, 2003).

\section{Cusi Cusi}

Cusi Cusi se encuentra en la Puna Jujeña, a 3.800 m.s.n.m., en el departamento de Rinconada, en la región de la Cuenca Superior del Río Grande de San Juan (Figura 1).

Figura 1. Mapa del área de estudio.
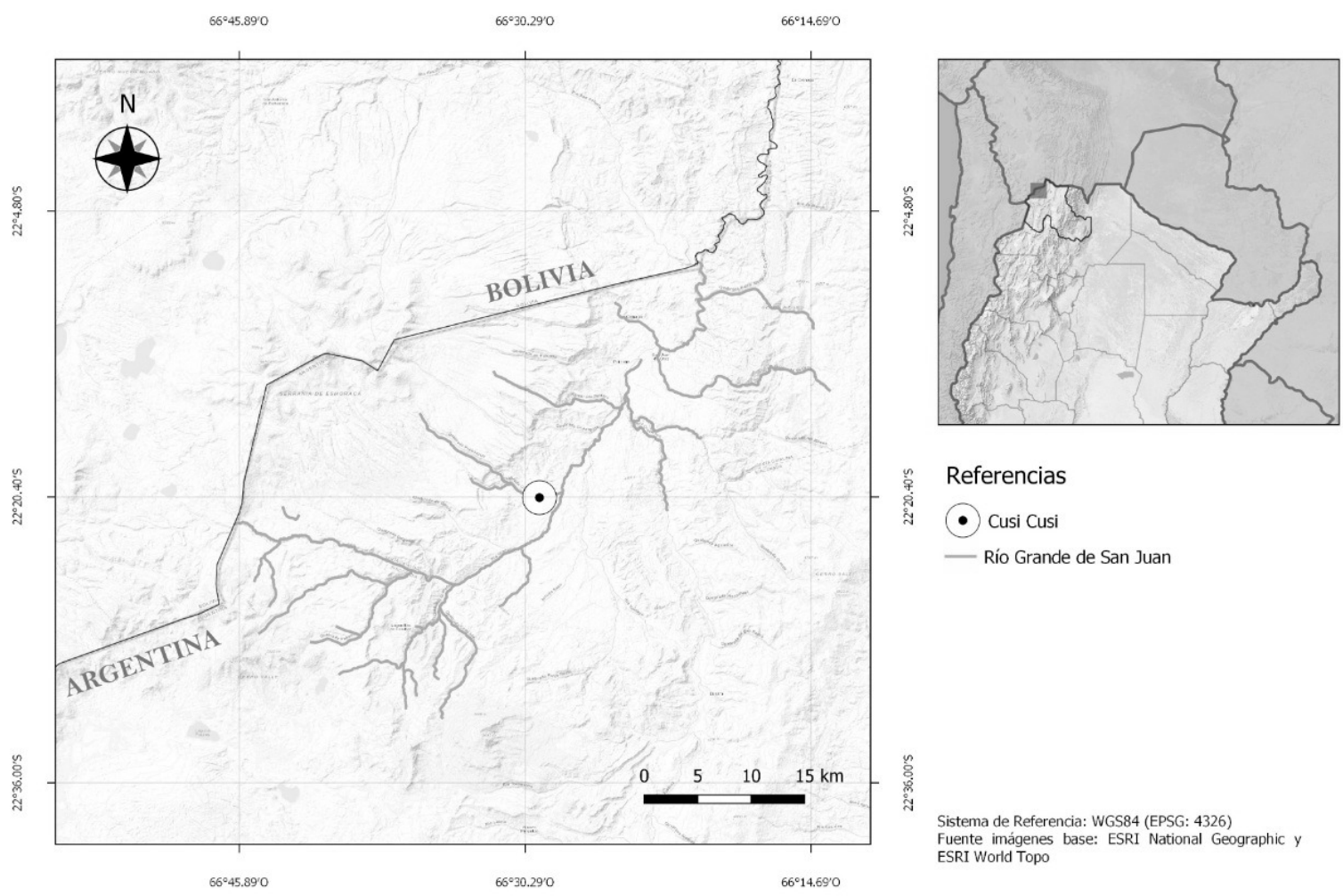

Fuente: Elaborado por Laura Pey. 
Es la localidad de mayor tamaño en la región y cabecera del municipio homónimo que incluye a las localidades de Misa Rumi, Ciénega de Paicone, Paicone y Lagunillas del Farallón. Según el Censo INDEC 2010, en el pueblo habitan 243 personas, aunque considerando la alta movilidad, es probable que el número sea mayor.

Al ser cabecera del municipio, el pueblo presenta instituciones estatales, comunales y religiosas. Cuenta con una Comisión Municipal, un jardín de infantes, una escuela primaria y un colegio polimodal, junto con una delegación policial y un centro de integración comunitario (C.I.C.), donde funciona la unidad sanitaria. A partir de 2015, la comisión vecinal se convirtió en la Comunidad Aborigen Orqo Runas, con personería jurídica y reconocimiento estatal y cuyas autoridades son elegidas por votación cada dos años por los miembros de la comunidad. El pueblo cuenta también con una iglesia católica apostólica romana y cinco iglesias evangélicas.

La región donde se encuentra emplazado Cusi Cusi fue parte de Bolivia hasta el año 1938, cuando se implementó el tratado de límites Carrillo-Díez de Medina planteado en 1925. Se trataba de una zona pastoril donde los habitantes vivían en caseríos aislados en un sistema de puestos y estancias donde se movilizaban con el ganado (Göbel, 2002; Carreras, 2015). Antes de la fundación del pueblo, Cusi Cusi era un lugar de reunión a donde viajaban las distintas familias para ocasiones festivas en lo que hoy se conoce como la "Iglesia Vieja". La actividad tradicional del pueblo es el pastoreo de llamas. Sin embargo, cada vez menos personas tienen esta forma de vida. De acuerdo con las entrevistas que realizamos, los adultos expresan su preocupación sobre el hecho de que a las generaciones más jóvenes no les interesa la "vida de campo".

\section{Desarrollo}

\section{La episteme local y las categorías de tiempo y espacio}

Las narrativas locales presentan el pasado dividido en dos períodos: el tiempo de los chullpas o antiguos y el tiempo de los abuelos. Los primeros pertenecen a un tiempo donde no existía el Sol. Los chullpas eran gente de pequeña estatura y construían sus casitas de piedra que ubicaban en las peñas y lugares de difícil acceso. Sobre su desaparición hay dos variantes, según los relatos: su muerte se produce con la llegada del Sol o con el Diluvio. Actualmente, en el paisaje han quedado evidencias de las casas y ciudades de los chullpas o antiguos, en lo que localmente se conoce como antigales o chullperíos. Estos espacios son considerados lugares peligrosos. Si uno los transita, debe tratarlos con respeto. Si se encuentran los huesos de los chullpas, estos no deben tocarse, ya que pueden producir distintas enfermedades y, en casos más extremos, la muerte (Vilca, 2009; Bugallo \& Vilca, 2011; Cruz, 2012):

\begin{abstract}
Se han muerto todos ya. No sé qué antigal sería pero yo he visto así en una peña grande hay rinconcitos también tirados con sus cuenquitos marroncitos. Así, todo tirado así, pero que no se podía entrar mis abuelos sabían decir, ahí hay chullpas sabían decirme, ¿ve? Entonces yo como era chica, era curiosa, entonces ir peña por peña y subir ahí, ahí tiene una casita también bonita la casita tiene, ahí están sus tejidos, sus ollas, sus cantaritos, todavía, ¿ve?... sus casitas sí, he visto, chiquita la casita, no es grande, y chiquitita la puerta donde mira y ahí estaban, sus ollitas, sus palitos, sus tejiditos que tejían. (Entrevista realizada a D. M.)
\end{abstract}

Las categorías nativas chullpa, antiguo, antigal, son demostraciones lingüísticas de las clasificaciones con las que nuestros interlocu- 
tores interpretan la temporalidad en su "mundo de la vida". Para los cuseños, el tiempo de los chullpas o antiguos no se puede ubicar en una fecha del calendario. Solo se sabe de ellos a través de los relatos de los abuelos y los restos materiales presentes en el paisaje. Consideran que no hay una relación de continuidad y parentesco entre ellos y los antiguos. Como ya se ha descrito en un trabajo anterior (Vaquer et al., 2020), el mito de los chullpas se ha registrado, con variantes locales, en otros contextos etnográficos a lo largo de la región Circumpuna (ver, por ejemplo, Sendón, 2010, y Cruz, 2014). A pesar de las diferencias, se pueden encontrar elementos comunes con la cosmovisión aymara y quechua que nos permiten ahondar en las concepciones de temporalidad de este tiempo.

Bouysse-Cassagne y Harris (1988) relacionan el mito de los chullpas con la segunda edad o pacha, que se encuentra asociada a lo salvaje y lo virgen. Los chullpas son los seres que habitaban en esta edad y que no tienen relación con la humanidad actual. Cuando sale el Sol, los chullpas se disecan y se vuelven charqui. En otras versiones, los chullpas mueren por el Diluvio. Por eso se los encuentra en sus "casitas" con sus cosas. Esta edad se encuentra vinculada con los cerros, lugares marginales de los centros agrícolas. Si bien los chullpas desaparecen con la salida del Sol, siempre existen relatos sobre alguno que queda vivo en lugares considerados marginales. Representan el extremo no social de una sociedad pensada en términos de parentesco, la alteridad extrema (Cáceres Chalco, 2016).

Sin embargo, los chullpas no desaparecieron. Además de los relatos sobre los "últimos chullpas", estos seres se encuentran en el ukhu pacha, el "mundo de abajo". En cualquier momento puede ocurrir un pachakuti, o inversión del pacha, y los lugares ontológicos pueden ser invertidos. El tiempo mítico andino permite que a través de los rituales se vuelva al tiempo originario y al espacio originario. Por lo tanto, el tiempo-espacio andino es reversible y escapa de la lógica del principio de causalidad y no contradicción. Tanto en aymara como en quechua el término pacha significa, indiferenciadamente, tiempo y espacio (Bouysse-Cassagne \& Harris, 1988). Esta espacialidad del mito es lo que permite vincular a la temporalidad con el paisaje. En consecuencia, si bien el mito no tiene una ubicación temporal lineal, sí tiene lugares específicos del paisaje donde se activa.

El tiempo de los abuelos comienza con la fundación del pueblo de Cusi Cusi. A diferencia del tiempo de los chullpas, esta narrativa se ubica en la temporalidad lineal y cronológica occidental. Si los chullpas representan la alteridad, los abuelos son los antecesores con quienes los comunarios reconocen relaciones genéticas. De hecho, muchas de las familias cuseñas son parientes de los fundadores del pueblo. Este momento se relaciona también con la forma de vida pastoril. En el tiempo de los abuelos, Cusi Cusi se incorpora a la historia nacional e internacional, con eventos lineales y sucesivos que se pueden ubicar en el tiempo y el espacio.

Cusi Cusi se funda alrededor de 1925 en lo que hoy se conoce como "Pueblo Viejo", donde vivían unas 5 o 6 familias y donde se construyó la "Iglesia Vieja" o "Capilla Vieja". Luego, en 1954, se realiza una segunda fundación en donde se encuentra actualmente emplazado el pueblo: 
[...] claro pues que nacido poco chico ya se ha criado el pueblo, poquito unas casitas, había dos, tres casitas, había registro civil, había estaba este correo, había policía, escuelita bajita techo con pajita la escuela. Y se ha formado más más más más se ha formado. (Entrevista realizada a D. M.)

El pueblo surge como un lugar de reunión para las familias de pastores que vivían dispersas en la zona y de lugares más lejanos en Bolivia. La fecha de la reunión es el 3 de mayo, actualmente conmemorada por los comunarios como momento de la fundación del pueblo. También coincide con la fiesta de la Santa Cruz, del Señor del Milagro, y con la fiesta prehispánica de la Chaqana, cuando las Pléyades son visibles en el cielo. Esta fiesta es dividida en dos: la primera parte, vinculada con la Santa Cruz, es celebrada por los comunarios católicos, ocasión en que se realizan actividades tradicionales, como el baile de la cuarteada y la preparación y consumo de la kalapurca (al respecto, ver Carreras \& Pey, 2019). Aquellos comuneros que adscriben a la religión evangelista no participan de esta celebración. La segunda parte de la fiesta es la conmemoración de la fundación de la Comisión Municipal, donde participa todo el pueblo. El nombre del pueblo, en idioma quechua, significa "alegría alegría", por dos razones: por la presencia en la región de un arácnido de igual nombre que, cuando aparece, representa un buen augurio y, también, como nos cuentan en las entrevistas, por esta identificación del pueblo como un lugar de encuentro y fiesta:

También era muy notorio, era grande, porque hay quien había en la capilla. Estaba arriba. Antes venía de todos lados la gente, pero yo creo que no había iglesias por todos lados, esta era la iglesia única, todos los pueblos concentraban ahí, era una juntada muy numérica. Mucha gente. Más que nada era más se juntaban las mujeres que formaban doctrinas, cantaban todo, era distinto. (Entrevista realizada a S. M.)
Preguntar nomás a todos como fue la, más, digamos a las personas mayores que fueron primero los que fundaron el pueblo, preguntarles a ellos como fue acá todo así, pero como aquí no fue Cusi Cusi, fue arriba en la capilla era el primer Cusi Cusi, y todo es bueno, preguntarle a la gente que eran de hace años. Ellos más saben. (Entrevista realizada a N. C.)

Esta época es valorada de maneras distintas por los entrevistados. En general, se acuerda con que antes había un mayor "respeto al medio ambiente", relacionado con las prácticas pastoriles. Aquí se evidencian ciertos lazos que unen el presente y el pasado. Lo espacial y lo material perduran estableciendo una continuidad temporal. A su vez, el ambiente es entendido tanto temporal como espacialmente. Los que hoy en día son pastores exaltan estos valores:

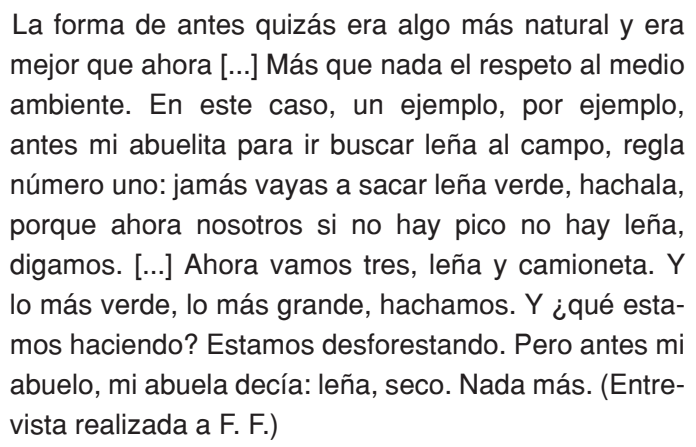

También quienes son agricultores expresan su preocupación por los cambios en el medio ambiente. El cuidado del ambiente refiere no solo a las prácticas productivas, sino también a prácticas rituales de cuidado, como el "dar de comer a la tierra" y la challa. Algunos continúan con esas tradiciones, vinculando así el pasado y el presente, mientras que otros no. Una pareja de evangélicos afirmaba en las entrevistas realizadas que no hay continuidad entre presente y pasado, justamente porque 
ellos no creen en la Pachamama ni en challar la tierra. La narrativa evangélica reescribe el pasado, ya que entra en contradicción con las historias de los abuelos. Genera una desvinculación entre pasado y presente y se produce un alejamiento a partir de la prohibición de determinadas prácticas en el paisaje. Dice L. A.: "parece que lo hubiéramos dejado atrás el pasado". Y continúa, sobre los chullpas:

\footnotetext{
Y a mí me contaron mis abuelos, me contaron del que ellos no conocían el sol ni la luna pero cuando había diluvio había el sol, la luna, existía ya, existía. Pero mis abuelos me dijeron que ellos no conocieron ni sol, solamente vivían del luna. Sí, así me decían mis abuelos, pero después que ahora estoy ya grande veo, comparo un poco con la Biblia pero no, me parece que no. Había sol y la luna, había ya. Pero no sé, pero tengo un poco de lo que yo pienso, no de lo que mis abuelos dicen [...]. Así es, yo también soy evangelista [...]. Yo le recuerdo de lo que dijeron mis abuelos, pero yo ya no lo creo tampoco, y no tengo miedo tampoco de que me va a hacer mal, nada nada. Yo no tengo miedo, hay lugares peligrosos hace años decían que hay lugares peligrosos que la tierra es mala, todo eso, pero no, yo ya no creo, pero hay gente que teme de eso. (Entrevista realizada a L. A.)
}

Del mismo modo, otras prácticas también se vinculan con la continuidad del pasado en el presente, como el idioma quechua, que muchos insisten en recuperar. También la Señalada, la fiesta patronal y el carnaval, eventos sociales que cada vez cuentan con menos asistentes, sea porque la gente se va del pueblo o porque no están permitidos por la religión. Otros valores fundamentales del modo de vida del pasado eran el respeto y el trabajo. Todos coinciden en que antes había mucho más esfuerzo. Según F. F.: "siesta para ellos era pecado directamente". Para los entrevistados más jóvenes, estos son aspectos que se deberían recuperar, mientras que para los abuelos que aún siguen vivos y
Ilevaron ese estilo de vida, fueron tiempos muy duros y de mucho sufrimiento.

En cuanto a los modos de acceder al pasado, los entrevistados alternan sus respuestas entre "a través de técnicos y arqueólogos" y "a través del relato de los abuelos". A veces se reconocen ambas vías. Por lo general, aquellos que llevan una vida más cercana a los valores tradicionales hacen más énfasis en las historias transmitidas oralmente. La mayoría hizo referencia a su infancia, remontándose a su propia experiencia pasada para pensar el pasado. Se pueden identificar ciertas jerarquías en el acceso a estos saberes: los mayores saben más que los jóvenes, y los hombres saben más que las mujeres. Tanto es así que varias de las mujeres entrevistadas afirmaban "no saber nada", si bien luego en el transcurso del diálogo demostraban conocer historias pasadas. Por otro lado, aquellos que están más vinculados a la vida política del pueblo respondieron que el modo de acceder al pasado es a través de la arqueología, de documentales y de libros, es decir, acercándose a la transmisión escrita y científica del conocimiento.

Por último, se habló también de para qué sirve conocer el pasado. Esta pregunta estipula una relación entre pasado y futuro. Una señora contestó: "El pasado ya sirve y abre el presente para ver las tres cuestiones: pasado, presente y futuro" (entrevista realizada a M. L.). En general, notamos la proyección de los deseos de cada entrevistado en el pasado, de aquello que les gustaría que haya hoy en día, y de allí la importancia de recuperarlo, conociéndolo y revalorizando las prácticas antiguas. Hay acuerdo en que "sirve para mostrar lo que se vivía y lo que se puede vivir en la zona", es decir, que conocer el pasado es útil para 
proyectar el futuro. Uno de los entrevistados hizo énfasis en querer dar a conocer la historia de Cusi Cusi para "que reconozca todo el país, que se identifique dónde está Cusi Cusi digamos", es decir, que se ubique geográficamente (entrevista realizada a R. Q.). Relaciona así lo temporal (el conocimiento del pasado de Cusi Cusi) con la espacialidad, y en específico con el espacio nacional. Tener una historia es tener un lugar, y formar parte del territorio es formar parte de la historia nacional.

De modo similar, uno de los abuelos expresó su deseo de querer "dejar para los que vienen por atrás", es decir, para los jóvenes (entrevista realizada a L. F.). Nuevamente lo temporal es expresado espacialmente, y, en este caso, el futuro es representado como viniendo desde atrás, y no hacia adelante. Resulta interesante, en este sentido, recuperar lo que la socióloga Silvia Rivera Cusicanqui (2015) plantea como el método qhipnayra (futuro-pasado). Según ella, el aforismo aymara qhipnayra uñtasis sarnaqapxañani, que podríamos traducir como "mirando hacia atrás y adelante (al futuro-pasado), podemos caminar en el presente-futuro", nos permite alejarnos de la linealidad temporal occidental para dar cuenta de cómo en el pensamiento andino el pasado y el futuro se encuentran contenidos en el presente.

\section{Categorías del espacio y el tiempo en las epistemes científicas}

El carácter objetivista de la ciencia occidental implica que ciertas categorías sean vaciadas de sus contenidos subjetivos. En particular, las categorías de tiempo y espacio, que son vectores fundamentales en la vida cotidiana, reciben una reformulación en el mundo de la ciencia. Siguiendo a Bourdieu (2003), consideramos que la ciencia es un campo social conformado por la lucha por el capital simbólico. En consecuencia, la visión de la ciencia que presentamos a continuación representa el punto de vista ortodoxo, lo cual no quita que existan otras visiones del tiempo y la temporalidad que la discutan. De hecho, nuestra propuesta forma parte de la heterodoxia que cuestiona la visión hegemónica.

La ciencia se apoya en el "mundo de la vida", pero opera a partir de una abstracción del mismo que termina negando su origen (Husserl, 2008). Es decir, sus categorías se consideran "objetivas", lo que niega su carácter construido e histórico. En el terreno antropológico, resulta interesante el aporte de Iparraguirre (2011, p. 45) para distinguir el tiempo como un hecho físico, o "fenómeno del devenir", y la temporalidad como la "aprehensión del devenir", esto es, social y culturalmente mediada. Así, la fórmula que introduce el objetivismo es introducir una noción de temporalidad como universal, reemplazándola por el tiempo. Este hecho es más notorio en el caso de la arqueología, ya que tiempo y espacio son categorías analíticas que utilizamos en nuestras interpretaciones del pasado.

Varios autores se dedicaron a la crítica de las categorías temporales que utiliza la arqueología (Shanks \& Tilley, 1987; Lucas, 2005; Jones, 2007). Una primera distinción que realizan es que no es lo mismo el tiempo que la cronología. La cronología implica un ordenamiento de fechas en el tiempo, y a diferentes escalas espaciales. El principio de causalidad es el que rige el orden y las secuencias: en el ordenamiento cronológico de fechas, fases y períodos se supone que los eventos más tempranos son la causa de los más tardíos. En muchos casos, 
la explicación arqueológica consiste en realizar los nexos causales entre los eventos que aparentemente se encuentran aislados.

Un ejemplo propio nos puede ilustrar: en las excavaciones de uno de los sitios en Cusi Cusi, Casas Quemadas, recuperamos restos de vaca (Bos Taurus) (Vaquer, 2016). En un primer momento consideramos que el sitio era prehispánico. La presencia de fauna hispana nos llevó a cuestionar esta interpretación y a preguntarnos sobre su ingreso al registro. Para ello, consultamos materiales etnohistóricos que trataban sobre los circuitos mercantiles coloniales, la necesidad de carne para proveer a los trabajadores de las minas y el traslado de ganado hacia lugares de matanza. Se estableció, así, una cadena causal:

invasión española $\rightarrow$ desestructuración de las poblaciones originarias $\rightarrow$ demanda de carne $\rightarrow$ circuitos arrieros de ganado.

Todos los eslabones de la cadena son eventos cronológicos que podríamos situar en fechas más o menos exactas. Ordenar estos eventos cronológicamente puede ser considerado como una explicación de la presencia de restos de vaca en el sitio. A su vez, ordenar los eventos en cronologías implica una linealidad, que tiene como presupuesto el principio de no contradicción. Según la formulación aristotélica, que es la base de la lógica tradicional, un ente no puede recibir predicados opuestos al mismo tiempo. El tiempo como movimiento se encuentra presupuesto en el principio de no contradicción. Al desarrollar la línea de tiempo de la cronología se establece una relación entre el pasado, el presente y el futuro que implica que los eventos considerados pasados, además de ser la causa de los eventos presentes, ya no están más. El principio de no contradicción sirve como guardián y ordenador de la cronología: lo pasado ya pasó, por eso no puede coexistir con lo presente. Si el pasado estuviera presente se produce una contradicción, ya que un ente tendría predicados distintos al mismo tiempo.

Esta visión del tiempo como cronología ha sido criticada ampliamente. Sin embargo, es la que prima en el sentido común de la interpretación arqueológica. La arqueología positivista descansa en la separación entre el pasado y el presente, entre el contexto sistémico y el contexto arqueológico; y la explicación arqueológica consiste en construir puentes metodológicos que permitan insuflar vida en los restos arqueológicos muertos del pasado (Schiffer, 1972; Binford, 1998). Así, se construyen modelos explicativos del pasado, que en ningún momento aceptan la instancia hermenéutica de la ciencia. Se jerarquiza el discurso científico, ya que, si estamos explicando una realidad, no queda lugar para otras explicaciones posibles, contradictorias y simultáneas de ese pasado. Esta consideración del tiempo arqueológico como cronología es la que sedimentó en la visión social de la arqueología. La arqueología ordena eventos en el tiempo y cuenta con herramientas (dataciones absolutas y relativas) para determinar la secuencia entre ellos. Somos ordenadores del pasado, esa es la función que nos reclaman socialmente: es la condición de verdad del discurso científico sobre el pasado.

Existe, por lo tanto, una división entre la teoría y la práctica arqueológica. Si bien se han realizado numerosas críticas a los supuestos modernos de nuestra disciplina, esto no implicó un cambio significativo en la práctica profesional. Mucho se ha escrito sobre la oposición 
entre naturaleza y cultura, sobre los dualismos cartesianos y modernos que cruzan nuestras interpretaciones. Pero el problema es que no es posible una superación de los mismos en el método (Vaquer, 2018). Para hacer arqueología, una etapa ineludible es la clasificación y la medición, la cronología y el análisis espacial. Mientras sigamos haciendo lo que hacemos, estos dualismos, estas objetivaciones no son posibles de superar. Lo único que nos queda es reconocerlas y aceptarlas como parte constitutiva de nuestras lógicas científicas, y que las clasificaciones y mediciones no sean el objetivo último de las interpretaciones del pasado. Y, sobre todo, entender que nuestras narrativas del pasado, construidas desde una lógica moderna y científica, son una narración más entre las tantas posibles. De esta manera, reconocemos el rol político y social de la arqueología y sometemos las conclusiones y los métodos a un choque constante con las lógicas de los seres humanos que habitan, en el presente, ese pasado que se pretende interpretar.

\section{Diálogos entre las epistemes: alcances y limitaciones}

Con fines analíticos, consideramos que existe una lógica con respecto a cada una de las narrativas cuando, de hecho, hay una variabilidad intrínseca. Esta variabilidad depende, en gran medida, de los campos desde los que se legitima el conocimiento sobre el pasado. En particular, identificamos tres fuentes de legitimación del conocimiento en Cusi Cusi: los abuelos y la forma tradicional en la que se hacen las cosas vinculada con la forma de vida pastoril; la Biblia, en el caso de los evangélicos, que opera sobre todo a partir de negar la lógica anterior; y las explicaciones científicas que generamos quienes estamos trabajando en la zona. Cada una de ellas se inserta en un campo global en el cual, desde la introducción del evangelismo, se produce una lucha por la ortodoxia, sensu Bourdieu (1977).

Interpretamos la composición de este campo en términos de tres variables que se combinan. La primera de ellas es la edad. Dividimos a los entrevistados en tres grupos etarios con relación a su valoración del pasado. El primer grupo está conformado por los jóvenes menores de 30 años; el segundo por la gente entre 30 y 60 años; y finalmente los mayores de 60. La segunda variable es la religión. Por un lado, los evangélicos, que tienen una visión negativa del pasado y pretenden abandonar las prácticas tradicionales porque las consideran "idolatría" (Segato, 2005). Por otro lado, están los católicos apostólicos romanos que continúan con las prácticas tradicionales. La tercera variable es el género. Las mujeres, si bien poseen, en la mayoría de los casos, experiencia directa de la vida pastoril, dicen no conocer nada (Vaquer et al., 2020). Algunas narrativas sobre el pasado, en particular las de orden discursivo, parecieran a simple vista ser patrimonio de los hombres. Esta variable deberá ser profundizada en trabajos futuros que adopten una perspectiva de género para poder dar cuenta de las relaciones de los géneros entre sí y con el pasado.

La lucha por el poder simbólico se da mayormente a partir de dos variables: la edad y la religión. Con respecto a la edad, los entrevistados de la franja entre 30 y 60 años son los que se apoyan en los abuelos y en la ciencia como fuentes de legitimación. Este grupo etario se corresponde con una generación "bisagra", ya que muchos de ellos experimentaron directa- 
mente la vida pastoril, pero también las modificaciones que implica la vida capitalista. Ellos son los que incorporan las explicaciones científicas como fuente de legitimación, ya que están interesados en explotar la región turísticamente. Sostienen que los turistas están interesados en los discursos científicos, tanto desde la arqueología como desde otras disciplinas. Este grupo es el que está disputando el poder en la comunidad, a partir de la creación de lugares alternativos a los tradicionales.

Con respecto a la religión, la lucha simbólica se da a partir de las representaciones del pasado. Los evangélicos tienen una postura más dura y condenan las prácticas tradicionales. Este proceso lo podemos interpretar en términos de las relaciones entre las narrativas religiosas y el "mundo de la vida", ya que las narrativas basadas en la Biblia proponen una reinterpretación radical de las prácticas tradicionales que conforman el "mundo de la vida" de los cuseños. La Biblia aparece como un texto ejemplar que legitima y establece un nuevo código de conducta que, a su vez, refuerza una concepción particular del pasado.

Por el lado de las narrativas científicas, tampoco podemos generalizar. Las posiciones en el campo académico son la principal variable de legitimación de las narrativas. Si bien en la práctica democratizamos las interpretaciones del pasado, la palabra del director del equipo siempre tiene más valor que la del resto de los integrantes. Esto cambia a medida que los diferentes miembros se van especializando en temas determinados, con lo que construyen lugares particulares de legitimación dentro del equipo y del campo académico en general (ver Vaquer, 2013, para un análisis más detallado del campo científico). Así, cada integrante del equipo comienza a construir su propia red de relaciones interpersonales con los miembros de la comunidad, por afinidades personales y temáticas.

Se produce una jerarquización de las narrativas en función de los diferentes contextos de enunciación. Al principio de nuestro trabajo era muy difícil que la gente hablara abiertamente sobre las narraciones tradicionales del pasado, ya que para muchos de ellos eran mitos y carecían de sustento. Una vez comenzado el proyecto del museo comunitario, la gente empezó a rescatar la forma de vida del pasado como una posible mercancía para mostrar a los turistas. Cuando alguno de la generación entre 30 y 60 años habla con nosotros, también hace referencia al conocimiento "científico", obtenido en diferentes cursos que se ofrecieron en la región. Lo mismo pasa con los evangélicos, dependiendo del contexto y del interlocutor acomodan su discurso para apelar a diferentes referentes de legitimación. Por lo tanto, existe una fluidez en las narrativas que escapa a todo intento simple de clasificarlas.

Ahora bien, las diferentes nociones de temporalidad se encuentran relacionadas con las distintas posturas enunciativas que presentamos anteriormente. Existen al menos tres temporalidades en la lógica cuseña. La primera es la temporalidad circular de la narración tradicional, donde el pasado, el presente y el futuro conviven en el paisaje. Este tiempo, relacionado con los chullpas, es un tiempo fuera del tiempo. Un segundo momento temporal se inicia con la fundación del pueblo. El acto fundacional del pueblo implica la introducción de una temporalidad lineal, que fue reforzada por instituciones como la Iglesia católica y la escuela. Finalmente, la tercera lógica temporal 
es la evangélica, que de acuerdo con Segato (2005), relaciona la historia de los pueblos puneños con el relato bíblico del "Pueblo Elegido". La narrativa bíblica refuerza también la concepción lineal del tiempo a partir del mito escatológico cristiano, que implica una creación y eventualmente un fin del mundo.

Con respecto a las relaciones entre la temporalidad y el "mundo de la vida", la introducción del evangelismo implica una reinterpretación del "acervo de conocimiento" tradicional, que ya había sufrido una reinterpretación a partir de la Conquista y la imposición del cristianismo. Se produce una dislocación entre los "mundos de la vida" representados por la forma de vida tradicional, el modo de vida capitalista y los nuevos modos de vida propuestos por el evangelismo. No obstante, esta dislocación es solamente aparente, ya que en la práctica las diferentes narrativas se acomodan a los distintos estados de situación.

Nuestras relaciones con la temporalidad también son complejas. Si bien cuando realizamos nuestras interpretaciones utilizamos el tiempo en cuanto cronología, y nuestra actividad académica así nos lo demanda, en nuestro "mundo de la vida" prima una noción más circular de la temporalidad (Husserl, 2008; Heidegger, 2012). Se produce una dislocación en nuestra relación con el tiempo: tenemos una relación con la temporalidad en nuestra vida cotidiana y otra en nuestra vida académica. Y a su vez, cuando tratamos con la gente de Cusi nos manejamos en términos híbridos, apelando a una u otra temporalidad en función del contexto y la situación de la enunciación.

Otro punto que nos parece importante clarificar es que las instancias dialógicas no se limitan solamente a la escritura de un trabajo científico. A los fines del ejercicio reflexivo sobre las narrativas arqueológicas, en este trabajo nos hemos enfocado en las narrativas que pertenecen al orden del discurso. Pero consideramos que existen otros momentos dialógicos con la comunidad donde se ponen en juego narrativas de orden práctico, específicamente en los trabajos etnográficos en Cusi Cusi, donde compartimos instancias dialógicas, como recorrer e interpretar el paisaje junto a miembros de la comunidad y participar de distintos eventos y fiestas.

\section{Conclusiones}

Desde la postura dialógica propuesta en este trabajo, vemos la diversidad en las lógicas interpretadas. El desafío que nos planteamos al comienzo fue realizar un diálogo entre las lógicas sin totalizar ni sintetizar, planteando una "visión crítica desde un locus existencial" (Wright, 2005, p. 70). Por un lado, tanto los cuseños como nosotros vivimos en un mundo capitalista globalizado. Esto acerca nuestras experiencias del "mundo de la vida", más allá de las diferencias particulares y los intereses, a veces contrastantes. Por otro lado, las particularidades del "mundo de la vida" cuseño escapan a nuestra experiencia urbana. Los acervos de conocimiento desde los que partimos son muy diferentes. Sin embargo, esto no implica la imposibilidad de generar un diálogo. Reconocer los puntos en común es, a nuestro entender, el primer paso para no exotizar a los cuseños y establecer una base común de trabajo.

También consideramos importante reconocer las diferencias, que al igual que los puntos en común, son producto de un acervo de conocimiento vinculado con condiciones diferentes de 
habitar. Las diferencias también son producto de los intereses particulares, que deben reconocerse en todo momento. Nuestro interés es laboral, aunque con los años las relaciones interpersonales van cambiando y se resignifican. Los intereses de los cuseños son más difíciles de sintetizar, ya que dependen de muchas variables y se modifican con el tiempo. Reconocer los intereses de los diferentes actores sociales (nosotros incluidos) es el primer paso para llegar a un diálogo.

Con respecto a las relaciones con la temporalidad, la variabilidad de posiciones impide también sintetizarlas en una sola. Son distintas lógicas que tienen como resultado varias narrativas sobre el pasado, que en muchos casos compiten entre ellas por convertirse en ortodoxia. El marco presentado nos resultó adecuado porque justamente permite dar cuenta de la variabilidad. Esta versatilidad de perspectivas, tanto para nosotros como para ellos, sirve como límite de las interpretaciones, tanto de un lado como del otro. Esto implica que en el campo de las narrativas sobre el pasado, si bien "juegan" todas las narrativas posibles, la episteme científica y la episteme cuseña se

\section{Referencias bibliográficas}

Binford, L. (1998). En busca del pasado. Barcelona: Crítica.

Bourdieu, P. (1977). Outline of a theory of practice. Cambridge: Cambridge University Press. (2003). El oficio de científico: Ciencia de la ciencia y reflexividad. Barcelona: Anagrama

Bouysse-Cassagne, T. \& Harris, O. (1988). Pacha: en torno al pensamiento aymara. En Albó, X. (Comp.), Raíces de América: El mundo aymará (pp. 217-281). Madrid: Alianza.

Bruner, E. (1986a). Experience and its expressions. En Turner, V. \& Bruner, E. (Eds.), The anthropology of experience (pp. 3-30). Chicago: University of Illinois Press.

(1986b). Ethnography as narrative. En Turner, V. \& Bruner,

E. (Eds.), The anthropology of experience (pp. 139-155). Chicago: limitan mutuamente. Esta idea de límite, de exterioridad de las lógicas entre sí, es la que permite que ninguna totalice a la otra, que no se conforme una sola narrativa en la que se diluyan las diferencias. La no totalización de la alteridad es la sutil diferencia entre el diálogo y la dialéctica (Levinas, 2002). Sin embargo, a medida que nuestro trabajo avanza, y ganan posiciones las posturas vinculadas con el turismo, la episteme científica es la que se consolida como fuente de legitimación de las narrativas sobre el pasado. El desafío que tenemos por delante junto a los cuseños es seguir manteniendo la diversidad de narrativas.

\section{Agradecimientos}

Queremos agradecer a la Comunidad Aborigen Orqo Runas y a la Comisión Municipal de Cusi Cusi por apoyar nuestros trabajos en la zona. También queremos agradecerles a todos los que se prestaron para las entrevistas por compartir sus puntos de vista con nosotros. A Pablo Wright, por los valiosos comentarios realizados al manuscrito. A Laura Pey, por la realización del mapa que acompaña este artículo.

University of Illinois Press.

Bugallo, L. \& Vilca, M. (2011). Cuidando el ánimu: salud y enfermedad en el mundo andino (Puna y Quebrada de Jujuy, Argentina). Nuevo Mundo Mundos Nuevos [En línea], Recuperado de http://journals.openedition.org/nuevomundo/61781.

Cáceres Chalco, E. (2016). Suq'as o hintilis, entre el orden y el desorden: la vida y la muerte en una misma dimensión en el sur andino de Perú. En Bugallo, L. \& Vilca, M. (Comps.), Wak'as, diablos y muertos: Alteridades significantes en el mundo andino (pp. 343371). San Salvador de Jujuy: IFEA, Ediunju.

Carreras, J. (2015). Fogones, hornos, cocinas y fuegueros de Cusi-Cusi (Puna de Jujuy): Análisis etnoarqueológico de las prácticas domésticas pastoriles vinculadas a las estructuras de 
combustión. (Tesis inédita de licenciatura). Facultad de Filosofía y Letras, Universidad de Buenos Aires, Buenos Aires.

Carreras, J. \& Pey, L. (2019). Explorando las formas de cocinar una kalapurca en Cusi Cusi (Puna de Jujuy): Etnografía de un plato andino. Antropologías del Sur, 6(12), 39-59.

Cruz, P. (2012). El mundo se explica al andar: Consideraciones en torno a la sacralización del paisaje en los Andes del sur de Bolivia (Potosí, Chuquisaca). Indiana, (29), 221-251.

(2014). Desde el diabólico mundo de los gentiles: Lecturas

sobre un pasado muy presente en el espacio alto-andino de Potosí y Chuquisaca (Bolivia). Revista Española de Antropología Americana, 44(1), 217-324.

Edgeworth, M. (2003). Acts of discovery: An ethnography of archaeological practice. Oxford: Archaeopress.

Göbel, B. (2002). La arquitectura del pastoreo: Uso del espacio y sistemas de asentamiento en la Puna de Atacama (Susques). Estudios Atacameños, (23), 53-76.

Guber, R. (2011). La etnografía: método, campo y reflexividad. Buenos Aires: Siglo XXI.

Heidegger, M. (2012). El ser y el tiempo. México: Fondo de Cultura Económica.

Husserl, E. (1982). La idea de la fenomenología: Cinco lecciones. México: Fondo de Cultura Económica.

(2008). La crisis de las ciencias europeas y la fenomenología trascendental. Buenos Aires: Prometeo.

INDEC (2010). Censo Nacional de Población, Hogares y Viviendas: Cartografía y códigos geográficos del Sistema Estadístico Nacional. Buenos Aires: Instituto Nacional de Estadística y Censos.

Iparraguirre, G. (2011). Antropología del tiempo: El caso mocoví. Buenos Aires: Sociedad Argentina de Antropología.

Jones, A. (2007). Memory and material culture. Cambridge: Cambridge University Press.

Levinas, E. (2002). Totalidad e infinito: Ensayo sobre la exterioridad. Salamanca: Sígueme.

Lucas, G. (2005). The archaeology of time. Londres y Nueva York: Routledge.

Rivera Cusicanqui, S. (2015). Sociología de la imagen: Miradas chi'xi desde la historia andina. Buenos Aires: Tinta Limón.
Schiffer, M. (1972). Archaeological context and systemic context. American Antiquity, 37(2), 156-165.

Schütz, A. \& Luckmann, T. (2009). Las estructuras del mundo de la vida. Buenos Aires: Amorrortu.

Segato, R. (2005). Cambio religioso y desetnificación: la expansión evangélica en los Andes Centrales de Argentina. En Guerrero Jiménez, B. (Comp.), De indio a hermano: Pentecostalismo indígena en América Latina (pp. 171-230). Iquique: Campus.

Sendón, P. (2010). Los límites de la humanidad: El mito de los ch'ullpa en Marcapata (Quispicanchi), Perú. Journal de la Société des Américanistes, 96(2), 133-179.

Shanks, M. \& Tilley, C. (1987). Social theory and archeology. Londres: Berg.

Turner, V. \& Bruner, E. (Eds.). (1986). The anthropology of experience. Chicago: University of Illinois Press.

Vaquer, J. M. (2013). La tradición como límite de la interpretación: Un ejemplo desde Cruz Vinto (Norte de Lípez, Bolivia). Relaciones de la Sociedad Argentina de Antropología, XXVIII(2), 269-291.

(2015). La arqueología como ciencia del espíritu: relaciones entre la arqueología, la hermenéutica filosófica y las consecuencias prácticas de las interpretaciones. Estudios Atacameños, 51, 15-32. (2016). La ocupación colonial temprana (S. XVI y XVII) en Casas Quemadas (Cusi Cusi, Rinconada, Jujuy): primeras aproximaciones a las relaciones entre lo local y lo global. Revista de Arqueología Histórica Argentina y Latinoamericana, 10(2), 1-26. (2018). Una descripción fenomenológica del objeto arqueológico. Chungará, 50(4), 623-632.

Vaquer, J. M., Petit, F. \& Di Tullio, M. (2020). Prácticas, narrativas y temporalidad en Cusi Cusi (Rinconada, Jujuy): una mirada hermenéutica. Andes, (31), 1-31.

Vilca, M. (2009). Más allá del paisaje: El espacio de la Puna y Quebrada de Jujuy: ¿comensal, anfitrión, interlocutor? Cuadernos de la FHyCS-UNJu, (36), 245-259.

Wright, P. (1994). Experiencia, intersubjetividad y existencia: Hacia una teoría-práctica de la etnografía. Runa, (XXI), 347-380. (2005). Cuerpos y espacios plurales: sobre la razón espacial de la práctica etnográfica. Indiana, (22), 55-72. 